\title{
La UAZ y el futuro de la educación superior de calidad
}

\section{RENÉ AMARO PEÑAFLORES*}

\author{
La Universidad Autónoma de Zacatecas, la institución pública de educación superior más relevante \\ de la entidad, ha atravesado por cuatro etapas históricas en las que se han configurado distintos \\ proyectos académicos y administrativos. Diversas investigaciones reflexionan en torno \\ de sus integrantes, proyectos, crisis, propósitos e impacto en la sociedad. Coinciden además \\ en que el conflicto de 1977 fue un acontecimiento clave en la formación de la universidad actual.
}

\section{Introducción}

Acerca de la historia de la educación superior en Zacatecas hay una importante relación de textos elaborada recientemente y cuyos resultados se hallan contenidos en libros, tesis, artículos y ponencias. Son varios los escritos con diversos enfoques teóricos y metodológicos realizados por académicos, investigadores, historiadores, sociólogos, estudiantes, profesores y analistas políticos. ${ }^{1}$ En efecto,

\footnotetext{
${ }^{1}$ Véase las siguientes referencias: Rosalina Ríos Zúñiga, «La creación de un plan de estudios moderno en el Instituto Literario de Zacatecas (1832-1854)», en Margarita Menegus (comp.), Universidad y sociedad en hispanoamérica. Grupos de poder siglos XVIII y XIX, México, Centro de Estudios sobre la Universidad-Universidad Nacional Autónoma de México/Plaza Valdés Editores, 2001, pp. 355-383; Rosalina Ríos Zúñiga, «La educación de la Colonia a la República: problemas y fuentes para el estudio de la transición», en Enrique González González y Leticia Pérez Puente (coords.), Colegios y Universidades. Del antiguo régimen al liberalismo, México, Centro de Estudios sobre la Universidad-Universidad Nacional Autónoma de México, 2001, pp. 89-103; María del Rosario Soto Lecasle, Actores educativos en la región minera de Zacatecas, 1754-1821, México, Universidad Pedagógica Nacional, 2007; José Luis Acevedo Hurtado, La Casa de
}

Estudios de Jerez: dos investigaciones (1832-1837), México, Universidad Autónoma de Zacatecas (Serie Va de nuez 2), 2008; José Luis Acevedo Hurtado, «Los estudios preparatorios en Zacatecas (1812-1850)», en René Amaro Peñaflores y María del Refugio Magallanes Delgado (coords.), Historia de la educación en Zacatecas I. Problemas, tendencias e instituciones en el siglo XIX, México, Universidad Pedagógica Nacional-Zacatecas, 2010, pp. 61-88; Norma Gutiérrez Hernández, «Mujeres que abrieron camino. La educación femenina en la ciudad de Zacatecas durante el porfiriato», tesis de Doctorado en Historia, Facultad de Filosofía y Letras de la Universidad Nacional Autónoma de México, México, 2012; Roberto Ramos Dávila, Haciendo memoria. Sintesis histórica de la Universidad Autónoma de Zacatecas, Zacatecas, Universidad Autónoma de Zacatecas (Serie Va de nuez 1), [1992] 2008; Froylán Casas Flores, «El gabinete de física del Instituto de Ciencias de Zacatecas», Digesto Documental de Zacatecas, núm. 9, vol. VIII, diciembre 2008, Zacatecas, Tribunal Superior de Justicia del Estado de Zacatecas, Maestría-Doctorado en Historia-Universidad Autónoma de Zacatecas, pp. 49-74; Froylán Casas Flores, «Experimentando con gabinetes. La educación científica en el Instituto de Ciencias de Zacatecas a finales del siglo XIX y principios del XX», tesis de Maestría en Historia, Maestría-Doctorado en Historia-Universidad Autónoma de Zacatecas, Zacatecas, 2010; Cuauhtémoc Esparza Sánchez, «José Árbol y Bonilla, un científico zacatecano», en Cuauhtémoc Esparza Sánchez (coord.), Zacatecas. Anuario de Historia, tomo II, Zacatecas, Centro de Investigaciones Históricas-Universidad Autónoma de Zacatecas, 1979, pp. 1131; Olivia Hernández Aguilar, «La educación superior a fines del siglo XIX. Planes de estudio en el Instituto de Ciencias de
*Docente
investigador
de la Unidad
Académica
de Historia,
Universidad
Autónoma
de Zacatecas 
existen estudios sobre la historia de instituciones como el Seminario Conciliar de la Purísima de Zacatecas, fundado en $1869,{ }^{2}$ o bien de las nuevas instituciones de enseñanza superior establecidas en Zacatecas a partir de la década de los 1970. ${ }^{3}$ De igual modo, del Instituto Tecnológico Regional de Zacatecas, fundado en 1976 con el objetivo de impulsar el desarrollo económico del estado; mientras que en 1985 se inauguró el Instituto Tecnológico y de Estudios Superiores de Monterrey (ITESM), Campus Zacatecas, que ha consolidado su estructura y

Zacatecas (1868-1904)», en René Amaro Peñaflores y María del Refugio Magallanes Delgado (coords.), Historia de la educación en Zacatecas I. Problemas, tendencias e instituciones en el siglo XIX, México, Universidad Pedagógica Nacional-Zacatecas, 2010, pp. 137-176; René Amaro Peñaflores, «Las escuelas de artes y oficios de Zacatecas durante el siglo XIX», en Francisco García González (coord.), Historia de la educación en Zacatecas: su enseñanza y escritura, Zacatecas, Universidad Pedagógica Nacional-Zacatecas, 2001, pp. 100-113; Hugo Ibarra Ortiz, El hospicio de niños de Guadalupe: educación, artes y oficios (1878-1928), México, Universidad Pedagógica Nacional-Zacatecas, 2009; Hugo Ibarra Ortiz, «La Escuela de Artes y Oficios de Guadalupe. Del porfiriato a la Revolución», en René Amaro Peñaflores y María del Refugio Magallanes Delgado (coords.), Historia de la educación en Zacatecas I. Problemas, tendencias e instituciones en el siglo XIX, México, Universidad Pedagógica Nacional-Zacatecas, 2010, pp. 177-225; Daniel Kuri Breña, El Instituto de Ciencias. Fenomenología de un plantel, México, Universidad Autónoma de Zacatecas (Serie Va de nuez 3), [1942] 2008; Francisco García González, «El difícil camino hacia la pluralidad en la Universidad Autónoma de Zacatecas», en Manuel Martínez Delgado (coord.), Pluralidad y universidad, Zacatecas, La Jornada Ediciones/Universidad Autónoma de Zacatecas, 1995; Francisco García González, Los días y los años de una institución. Historia contemporánea de la Universidad Autónoma de Zacatecas, México, Ediciones Cuéllar/ Universidad Autónoma de Zacatecas, 1997; Said Iván Cardoza Zúñiga y Adolfo Trejo Luna, «El movimiento universitario de 1977: ¿cambio o continuidad?», tesis de Licenciatura, Unidad Académica de Historia-Universidad Autónoma de Zacatecas, 2008; Said Iván Cardoza Zúñiga, «La autonomía universitaria: un redescubrimiento de su función en la Universidad Autónoma de Zacatecas», tesis de Maestría, Maestría-Doctorado en Historia, Unidad Académica de Historia-Universidad Autónoma de Zacatecas, 2011.

${ }^{2}$ Véase Martha Acosta Frías, «De la instrucción a la educación. La formación de las mujeres en el porfiriato zacatecano», tesis de Maestría en Humanidades, Área Historia, Universidad Autónoma de Zacatecas, 2008.

${ }^{3}$ Véase René Amaro Peñaflores, «En la búsqueda de una identidad. Pasado, presente y porvenir de la UPN Zacatecas», inédito. prestigio formativo empresarial en la entidad. ${ }^{4}$ Ambos tecnológicos representan importantes opciones educativas de enseñanza superior de carácter público y privado.

Al comienzo del siglo XXI, el crecimiento de la población amplió el mercado local de la educación superior tecnológica y comercial, con ello se diversificó la oferta a los sectores medios y la elite. A los sectores populares de varias regiones del estado se les dio respuesta a su potencial demanda con la instalación de varios institutos y universidades tecnológicas desde los 1990: Instituto Tecnológico Superior de Zacatecas, sede Norte (1990), sede Sur (1991), sede Fresnillo (1994) y sede Occidente (2000); además se fundó en Guadalupe la Universidad Tecnológica del Estado de Zacatecas (1998). El auge de tecnológicos regionales quedó regulado bajo el esquema estatal o federal, como organismos desconcentrados o descentralizados. ${ }^{5}$

En ese sentido, es preciso cuestionarse en torno a las enseñanzas, las experiencias y los conocimientos que se pueden recuperar de los estudios sobre la Universidad Autónoma de Zacatecas (UAZ) realizados en los últimos años a fin de reconfigurar y consolidar procesos formativos de calidad exigidos por la realidad presente y planteados por el futuro derrotero de la universidad pública. En adición, cabría preguntarse cómo recobrar el conocimiento histórico concerniente a la UAZ para explicar la problemática universitaria actual, la cual forma parte de un patrón común de la educación superior en México.

\footnotetext{
${ }^{4}$ Véase Alejandra Castañeda Pérez, «Educación superior, modernización y nueva Sociedad del Conocimiento en Zacatecas: Universidad Autónoma de Zacatecas e Instituto Tecnológico y de Estudios Superiores de Monterrey campus Zacatecas, 1968-2000», tesis de Maestría, Maestría-Doctorado en Historia, Unidad Académica de Historia-Universidad Autónoma de Zacatecas, 2012.

${ }^{5}$ Lourdes Consuelo Pacheco Ladrón de Guevara, «La legislación como obstáculo al cambio universitario», en Lourdes Consuelo Pacheco Ladrón de Guevara y Arturo Murillo Beltrán (coords.), 30 años de universidad. Lo que somos, lo que queremos ser, México, Universidad Autónoma de Nayarit, 1999, pp. 232-233.
} 
El objetivo de este trabajo es reflexionar $-\mathrm{a}$ partir del caso de la UAZ - sobre lo escrito recientemente en torno a la educación superior en la entidad y estructurar una explicación global de los procesos de institucionalización, consolidación, crisis, estabilidad, pluralidad, politización o despolitización de la vida universitaria. Se reconoce que hay estudios relevantes que han expuesto los momentos históricos más álgidos, las coyunturas (como la que permitió el tránsito del Instituto de Ciencias Autónomo de Zacatecas a universidad), el conflicto de 1977, su caracterización como universidad-pueblo, la historia de los procesos e imaginarios sociales que forjaron una identidad entre sus actores - estudiantes, maestros y autoridades - que a la fecha prevalece, y los problemas de la autonomía universitaria y las formas en que se ha vivido. ${ }^{6}$

Sin embargo, se asegura que hace falta esclarecer de modo detallado la conformación de un proyecto académico que mantuvo continuidad en el tránsito del Instituto de Ciencias Autónomo de Zacatecas (ICAZ) a la UAZ y que tras el conflicto de 1977 la cohesión y defensa de la autonomía universitaria se afianzó, para luego sumergirse en un periodo desgastante de peleas internas políticas e ideológicas. Asimismo, se requiere profundizar en el proyecto universidad-pueblo y en la aparición de un proceso de desinterés-apatía por la participación política y las luchas sociales, con la consecuente cancelación de la función crítica en la vida universitaria que perdura en la actualidad. Tal proceso complejo culminó a mediados de los 1990, cuando la UAZ, a pesar de avanzar en ciertos rubros académicos e investigativos, enfrentó una profunda crisis global, sobre todo financiera.

\footnotetext{
${ }^{6}$ Mariana Terán Fuentes et al., Diversas formas de vivir la autonomía universitaria. Reflexiones y experiencias, Zacatecas, Universidad Autónoma de Zacatecas/RHUEM/Consejo Mexicano de Investigación Educativa, 2010.
}

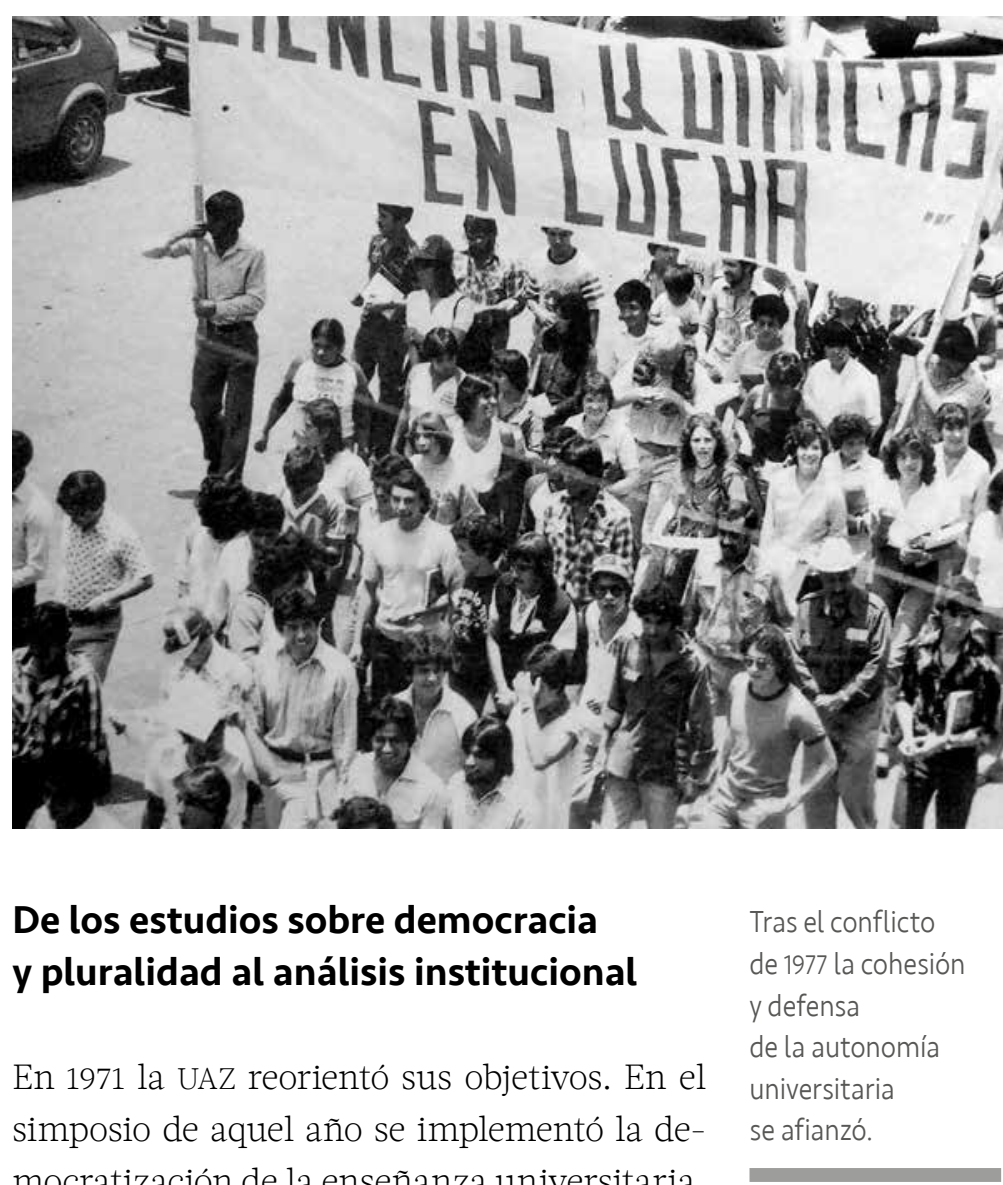

mocratización de la enseñanza universitaria, se adoptó un compromiso con las clases populares y se promovió el cambio social. Luego del conflicto interno de 1977, entre la derecha universitaria y los grupos progresistas, se consolidó el proceso de democracia y la autonomía, junto con la participación estudiantil y docente en las luchas populares; ello dio paso a la pluralidad, evidente tras el magno Congreso General Universitario (1987-1988). Dicha pluralidad le permitió a la institución conservar el diálogo con los grupos políticos opositores en la discusión y toma de decisiones que regían la vida cotidiana universitaria. Así se garantizaba la tolerancia, el respeto, la otredad universitaria.?

La pluralidad se fortaleció mediante el ejercicio de la cultura política forjada con las experiencias históricas de grupos y actores

${ }^{7}$ Manuel Martínez Delgado, «Antecedentes de la pluralidad en la Universidad Autónoma de Zacatecas», en Manuel Martínez Delgado (coord.), op. cit., pp. 51-76. 
universitarios. ${ }^{8}$ Así, la adopción de la pluralidad universitaria como vector ha facilitado que culmine el precepto: «El respeto al argumento del otro y del que piensa diferente». . No obstante, en los 1990 se percibió una comunidad universitaria «sumergida en la indiferencia y en el hastío discursivo». ${ }^{10}$ ¿Cuándo se perdió el afán de transformar a la universidad en un espacio que albergue distintas ideas?

Eduardo Remedi Allione ${ }^{11}$ realiza un acercamiento histórico con un análisis institucional, pues rastrea desde sus orígenes hasta su modernización en los 1990 y primeros años del siglo XXI. Dicha etapa osciló entre rupturas y construcciones sociales de los actores - maestros, autoridades y estudiantes-, de imaginarios y «fantasmas» que posibilitaron la formación de una identidad universitaria y mediaron en la vida institucional, política y académica. La institucionalización comenzó su desarrollo a mediados de 1930 para concluir el movimiento de 1977. De esa manera aparecieron trayectorias y quehaceres, espacios de lucha y defensa de la autonomía universitaria, así como contradicciones entre maestros y estudiantes, enfrentamientos con los gobiernos estatal y federal, incremento de la planta docente, masificación estudiantil y vinculación con la lucha popular. El cambio en la estructura de gobierno universitario, más democrática, respondió a modelos y construcciones de pensamiento, posiciones dirimidas al interior de la universidad.

Esta «historia global», sociológica y psicoanalítica, articuló los elementos de una identidad que cohesionó a la institución dentro de un marco coyuntural en el que las prácticas de

\footnotetext{
${ }^{8}$ Francisco García González, «El difícil camino hacia la pluralidad en la Universidad Autónoma de Zacatecas», p. 77.

${ }^{9}$ Manuel Martínez Delgado, op. cit., pp. 51-76.

${ }^{10}$ Luis Medina Lizalde, «Las singularidades de una pluralidad predicada», en Manuel Martínez Delgado (coord.), op. cit., p. 133.

${ }^{11}$ Eduardo Remedi Allione, Detrás del murmullo. Vida político-académica en la Universidad Autónoma de Zacatecas 1959-1977, México, Universidad Autónoma de Zacatecas/Juan Pablos, 2008.
}

los actores educativos, en los límites de la autonomía universitaria, configuraron un escenario específico, pero el autor presta más atención a la comprensión de los sujetos que a la evolución de la historia. La clave de la problematización es la recuperación de las identidades adscritas a lugares sociales. Se recuperan a los sujetos por épocas desde el ICAZ hasta el «proyecto utópico cargado de contenido igualitario», a raíz del conflicto de 1977, primero contra la derecha en defensa de la autonomía y el restablecimiento de la vida institucional, y luego contra Alianza Universitaria, facción que amenazaba la continuidad del proyecto de universidad democrática, científica, crítica y popular: imaginario esbozado por una red identitaria, solidaria y de lealtades sociolaborales.

Otro punto clave es la construcción de la autonomía institucional, que transita por una dialéctica de vínculos instituyentes e instituidos, así como nuevas relaciones sociales académicas; ello da pauta a la diversidad de posturas que presionaron, resistieron y consolidaron su oposición a los diferentes sentidos de los proyectos institucionales. Surgieron tensiones de micropolítica universitaria, contradicciones diversas, cuando se impuso paulatinamente la identidad institucional del grupo hegemónico: la izquierda institucional.

De cualquier manera, ese estudio es muy valioso en sus alcances teóricos y empíricos, aunque únicamente examina a profundidad el utópico e igualitario y deja de lado los imaginarios institucionales del otro «proyecto», el de la facción conservadora que buscaba neutralizar la participación política con sentido social e impedir, por tanto, la concientización social de los estudiantes universitarios. Por último, se impusieron nuevas visiones con el inicio de la profesionalización de la docencia en los 1980; la potencialidad estudiantil, tras la incorporación como docentes, devino en múltiples imaginarios, identidades y preocupaciones. Las dificultades académicas propias 
de quehacer docente y el acoso presupuestario permanente motivaron el desinterés y la ruptura del proyecto democrático; se diluyó la función crítica universitaria.

\section{Del análisis histórico a la historia-testimonios}

La reflexión histórica que explica los complejos procesos universitarios acaecidos de 1968 a 1992 se manifiesta en el trabajo de Francisco García González, que da cuenta de la transformación del ICAZ a UAZ y de las posteriores épocas por las que transita la vida universitaria, hasta arribar a los 1990, en los que «se intenta materializar el experimento de la pluralidad en la conducción de la universidad». ${ }^{12}$

Cabe resaltar que la historia contemporánea de la UAZ atraviesa por cuatro épocas. La primera (1986-1972) se caracteriza por un cambio de las formas de vida universitaria; significa el paso del atraso académico y del autoritarismo del gobierno universitario a relaciones democráticas, así como a una modificación en el estilo de asociarse con el gobierno y la «oligarquía» local. El primer Simpósium de Reforma Universitaria (19711972) definió el nuevo papel que debía ocupar la UAZ en la sociedad.

La segunda época (1972-1976) tuvo como rasgos importantes la democratización, el surgimiento de líderes sociales, la emergencia en la universidad de los jóvenes-estudiantes provenientes de los sectores populares. El resultado fue el «vínculo orgánico» entre maestros y estudiantes con los movimientos campesinos y de colonos; aunado a la aparición de un sindicalismo independiente y del Frente Popular de Zacatecas (FPZ). La democratización universitaria y su articulación sindical-popular generó que las relaciones con el gobierno local y los grupos opositores al proyecto universi-

\footnotetext{
${ }^{12}$ Francisco García González, Los días y los años de una institución.., p. 15.
}

dad-pueblo se tornaran conflictivas, de allí la ruptura violenta en 1977 (la «peor crisis política») y, al mismo tiempo, el momento que definió a la UAZ moderna. La defensa de la autonomía y el restablecimiento de la vida institucional tuvieron lugar hasta 1980.

Durante la tercera época (1980-1984) ocurrió el enfrentamiento interno entre diferentes grupos políticos, unos apoyados por el Partido Comunista Mexicano (PCM) y más tarde por el Partido Socialista Unificado de México (PSUM); surgió Tendencia Democrática Independiente, al igual que otros grupos de docentes y estudiantes apoyados por el gobierno estatal, aglutinados en torno a Alianza Universitaria.

La cuarta época (1984-1992) fue determinada por la armonía de los grupos políticos internos, ya que se abandonó la confrontación con el gobierno local y se estableció una política de aparente cordialidad. Se trató de un periodo de armonía-concertación-estabilidad que propició la organización del Congreso General Universitario (1987-1988), el cual confirmó la naturaleza democrática-crítica-popular y consolidó a la institución con el plan de desarrollo institucional. En la década de 1990, última fase de la época, uno de los propósitos

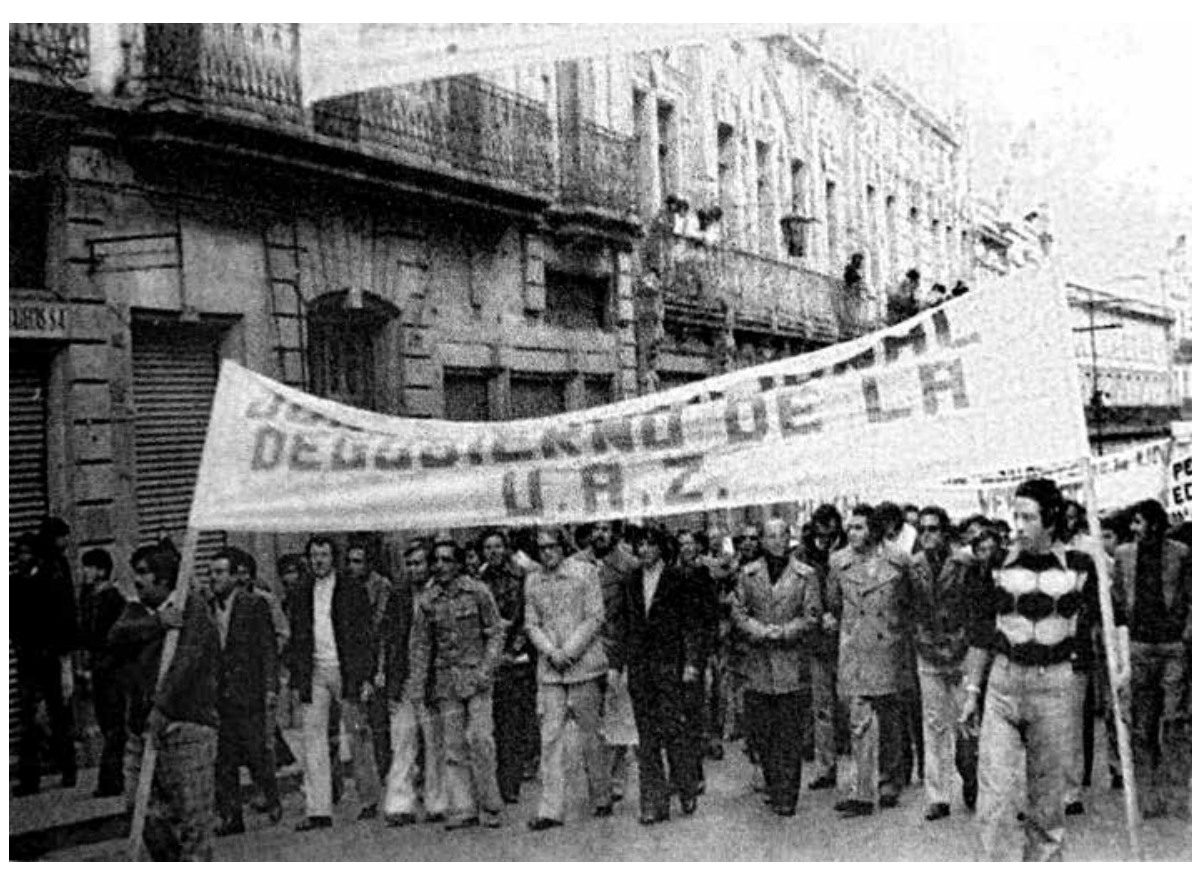




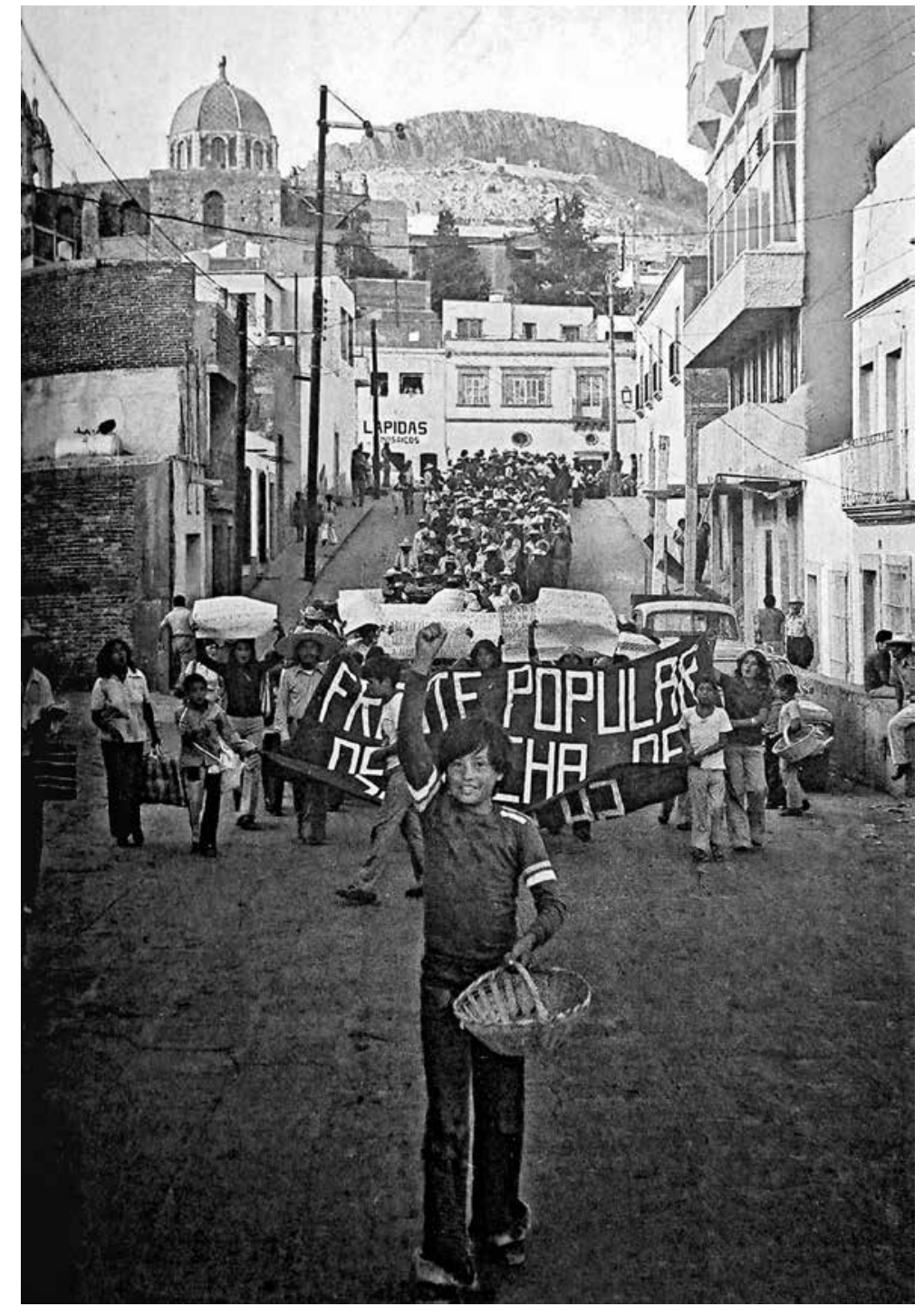

La experiencia de 1977 fue

fundamental, la UAZ se modernizó, se vinculó con

la sociedad y

marcó una ruta académica, cultural

y democrática.

Fotografías

tomadas de

Abel García Guízar

et al., op. cit. centrales era fortalecer la pluralidad como el precepto conductor de la UAZ.

En síntesis, García González brinda una pedagogía precisa acerca de la historia universitaria al enfatizar los logros más preciados de sus integrantes: los avances en la democratización, el crecimiento administrativo y académico, la ampliación de la cobertura educativa en los sectores populares y de la oferta curricular. En sentido crítico, se advierte:

Además de los elementos exógenos, los problemas internos han sido la traba fundamental en la maduración institucional (...) [pues] resaltan inercias negativas, obsolescencias y conducciones inco- rrectas que en gran medida explican la profunda crisis institucional que sufre actualmente la UAZ. ${ }^{13}$

Abel García Guízar et al. ${ }^{14}$ presentan testimonios sobre la faceta histórica más determinante, aquella que le planteó a la institución un punto de inflexión: cambiar para modernizarse o permanecer como corporación anclada a la tradición. Se basan en diversas reflexiones, que engloban elementos históricos y subjetivos, pues los autores fueron artífices del conflicto de 1977 (maestros, estudiantes y militantes). Pero tal subjetividad, compuesta de testimonios, recuerdos y memoria viva, triangulada entre sí con documentos y contexto, hace que emerja una intersubjetividad que le da sentido, verdad y objetividad a los acontecimientos de dicho momento histórico. Los ensayos que componen el libro se diferencian en cuanto a su nivel académico: unos se orientan hacia una narrativa histórica esencial, hacia el relato de ciertos pasajes del conflicto, y otros son más «académicos», apelan a una historia más analítica. Hoy, tales actores son académicos connotados, políticos respetados o figuras sobresalientes en el ámbito cultural. Todos configuraron un movimiento con un alto grado de identidad que facultó a la «adolescente universidad» a resistir los embates de la derecha universitaria, ante las determinaciones gubernamentales por preservar el control político y convertir a la institución en un espacio «neutral», alejado de las causas sociales-populares y de la izquierda partidista.

En la defensa de la UAZ, a partir de la lucha de 1977, los ensayos testimoniales son muy valiosos y complementan la reflexión histórica en forma académica y bien documentada.

\footnotetext{
${ }^{13}$ Rodolfo García Zamora, «Prólogo», en Francisco García González, Los años y los días de una institución, p. 11.

${ }^{14}$ Abel García Guízar et al., 1977. Autonomía y sociedad en Zacatecas. Una historia recuperada, México, Taberna Libraria Editores, 2011.
} 
Asimismo informan del conflicto más álgido, que comenzó tras la toma de la rectoría por un grupo de la derecha renuente al cambio y que buscaba derribar la cultura liberal y progresista. El grupo Alianza Universitaria se mostraba intolerante y preservador de la tradición, lo cual se oponía a las transformaciones que ocurrían desde 1971; combatía entonces la visión liberal, izquierdista y socializante que poco a poco, de acuerdo con su postura, invadía a la institución.

Ante los gobiernos estatal y federal, se encontraba en riesgo la autonomía universitaria y la independencia administrativa, financiera y curricular. La experiencia de 1977 fue fundamental, pues la vida pública local cambió radicalmente. La UAZ se modernizó, se vinculó con la sociedad y marcó una ruta académica, cultural y democrática. Si bien su transformación estructural con el tiempo posibilitó el tránsito de la universidad-pueblo, de la democracia a la modernización, también promovió la exclusión. El desgaste por las constantes presiones del Estado y la carencia permanente de recursos financieros, pese a la elevación de la autonomía a rango constitucional y la ley orgánica, generó una masificación - estudiantil, docente y de trabajadores - que repercutió en la calidad educativa. Las exigencias mediante evaluaciones y autoevaluaciones académicas, como mecanismos de control en los 1990, minaron la autonomía universitaria hasta convertirla en algo obsoleto. En la actualidad, la UAZ se ha convertido en una institución excluyente y reproductora de desigualdades sociales al margen de las necesidades del aparato productivo local y sin un proyecto de desarrollo integral.

De esa forma, el sector triunfante, el más comprometido con campesinos y colonos, promotores y dirigentes del FPZ, arribó a la rectoría universitaria (1980-1984). Sin embargo, no se flexibilizaron las estructuras académica y administrativa, aunque sí se intensificó la participación popular. Es cierto que se logró un avance hacia nuevas reformas universitarias, como la de 1987-1988 en la que se reivindicó a la universidad democrática, crítica y popular, y cuyos actores son los universitarios progresistas-populistas y los «progresistas-oficialistas» (aliados al gobierno estatal). Complementariamente, apareció un consenso que permitió implementar un plan de desarrollo institucional y la ulterior reforma universitaria de la década de 1990, acorde con las determinaciones impuestas por la globalización y los requerimientos del Estado neoliberal mexicano.

Nuevos problemas emergentes enfrenta hoy la institución: la proletarización agrícola local, el desempleo y la creciente migración hacia Estados Unidos. Así, «el reto y la ruta» para la UAZ es la elaboración de un proyecto académico y de investigación general vinculado al gobierno local, incluyente sectorialmente, que posibilite superar las deficiencias endémicas: las estructuras académicas y administrativas de los 1970 y 1980, la concentración de la matrícula estudiantil y los déficits crónicos financieros.

En suma, los trabajos que contiene este libro brindan un acercamiento a la problemática general de la UAZ, desde sus orígenes como heredera de las luchas estudiantiles de 1968; así como de los afanes de actores académicos, sociales y políticos, poseedores de una ideología marxista, socialista, liberal, progresista. Mediante la resistencia, la defensa y la organización social y popular, protegieron la autonomía, no como un precepto empleado por el gobierno local desde el cambio del ICAZ a la universidad, sino que pelearon por las causas populares con un proyecto académico que trascendió el espacio universitario hacia la sociedad zacatecana en las décadas de 1970 y 1980. El proyecto de universidad-pueblo devino en un inmovilismo administrativo y académico, y en la centralización de las decisiones universitarias que paulatinamente cancelaron 
el sentido crítico entre estudiantes y maestros, entre la universidad y la sociedad.

La cuestión de la autonomía universitaria, ${ }^{15}$ su preservación de forma directa o indirecta, se vislumbra en todos los ensayos del libro, pero se omite la calidad educativa (con excepción del texto de García Zamora). Es preciso comentar que ésta subyace en la urgencia de un proyecto de desarrollo integral ya discutido en las reformas de la «Universidad del siglo XXI» en los 1990; sin embargo, su postergación suprimiría la utopía universitaria, configurada con las luchas sociales de los 1970.

\section{Reflexiones finales}

Las investigaciones recientes relativas a la historia de la UAZ son de tipo sociológico-político (pluralidad), histórico-sociológico-institucional (imaginarios institucionales) e histórico-testimonial (recuerdos, relatos, memoria viva). Apuntan hacia un análisis histórico, si bien se centran en problemas y objetos de estudio carentes estrictamente de categorías históricas (cambios y continuidades, coyunturas y estructuras de larga duración). En efecto, el libro de García González ${ }^{16}$ constituye es una reflexión con un sólido sustento histórico, pues recurre al utillaje empírico y conceptual para explicar e interpretar los cambios ocurridos entre 1968-1992; asimismo, periodiza y caracteriza los procesos más relevantes sucedidos en las cuatro épocas con la intención de delimitar su espacio tempo-

\footnotetext{
${ }^{15}$ Véase Said Iván Cardoza Zúñiga, «La autonomía universitaria: un redescubrimiento de su función en la Universidad Autónoma de Zacatecas», tesis de Maestría, Maestría-Doctorado en Historia, Unidad Académica de Historia-Universidad Autónoma de Zacatecas, 2011; Alejandra Castañeda Pérez, «Educación superior, modernización y nueva Sociedad de Conocimiento en Zacatecas: Universidad Autónoma de Zacatecas e Instituto Tecnológico y de Estudios Superiores de Monterrey campus Zacatecas, 1968-2000», tesis de Maestría, Maestría-Doctorado en Historia, Unidad Académica de Historia-Universidad Autónoma de Zacatecas, 2012.

${ }^{16}$ Francisco García González, Los días y los años de una institución...
}

ral. Como historia global profundiza en acontecimientos y coyunturas universitarias, pero soslaya el aspecto comparativo.

Pese a lo anterior, dichos trabajos se acercan, a través de diversas disciplinas, a una explicación histórica valiosa, a la comprensión de la compleja historia contemporánea de la UAZ. Al respecto, coinciden en que la crisis de 1977 dio paso a ulteriores avances políticos, académicos y del sentido popular; no obstante, no se enfatiza que también se gestó la profunda crisis de los 1990. Entonces, está pendiente un análisis interdisciplinario y comparativo que haga factible explicar la manera en que el proceso incipiente de consolidación académica de finales de siglo hasta la actualidad es resultado de tales experiencias históricas: de continuidades y rupturas, de crisis económicas y políticas experimentadas por el Estado benefactor de los 1970 o los 1990, de estabilidad académica y logros, en los que la universidad se erige como referente de la educación superior local y regional, cuyo «proyecto integral» se dirige a su consolidación. 